\title{
5D World-Universe Model Space-Time-Energy
}

\author{
Vladimir S. Netchitailo \\ Biolase Inc., Irvine, USA \\ Email: v.netchitailo@sbcglobal.net
}

Received 17 June 2015; accepted 14 July 2015; published 17 July 2015

Copyright (C) 2015 by author and Scientific Research Publishing Inc.

This work is licensed under the Creative Commons Attribution International License (CC BY). http://creativecommons.org/licenses/by/4.0/

CC) (i) Open Access

\begin{abstract}
5D Space-Time-Energy World-Universe Model is a unified model of the World built around the concept of Medium, composed of massive particles (protons, electrons, photons, neutrinos, and dark matter particles). The Model provides a mathematical framework that enables precise calculation of medium-bound physical parameters: Hubble's parameter, intergalactic plasma parameters, temperature of microwave background radiation and the rest mass of photons. This paper aligns the World-Universe Model (WUM) with the theoretical framework developed by Prof. P. S. Wesson, albeit assigning a new physical meaning to the fifth coordinate. In the World-Universe Model, the fifth dimension is associated with the total energy of the Medium of the World, and the gravitomagnetic parameter of the Medium serves as the dimension-transposing parameter.
\end{abstract}

\section{Keywords}

5D World-Universe Model, Space-Time-Energy, Medium of the World, Intergalactic Plasma, Microwave Background Radiation, Mass Varying Photons

\section{Introduction}

We can't solve problems by using the same kind of thinking we used when we created them.

Albert Einstein

WUM is proposed as an alternative to the prevailing Big Bang Model of standard physical cosmology. The main difference is the source of the World's energy. In the present work, we focus on the physical meaning of the fifth coordinate and provide a brief overview of WUM.

World-Universe Model (WUM) utilizes the following principles:

Variable gravitational parameter. This hypothesis was proposed by Paul Dirac in 1937 [1].

Continuous creation of matter. Hoyle and Narlikar in 1964 offered an explanation for the appearance of 
new matter by postulating the existence of what they dubbed the "creation field", or just the "C-field" [2].

According to WUM, the World is a 3-sphere that is the surface of a 4-ball Nucleus of the World. The 4-ball is expanding in the 4-dimensional Universe, and its surface, the 3-sphere, is likewise expanding. The total surface energy of the 4-ball is increasing as it expands, thus creating new matter in our 3-sphere World.

Supremacy of matter was postulated by Albert Einstein: "When forced to summarize the theory of relativity in one sentence: time and space and gravitation have no separate existence from matter".

Existence of the Medium of the World was stated by Nikola Tesla: "All attempts to explain the workings of the universe without recognizing the existence of the ether and the indispensable function it plays in the phenomena are futile and destined to oblivion". Unique properties of the Medium were discussed by James McCullagh in 1846. He proposed a theory of a rotationally elastic medium, i.e. a medium in which every particle resists absolute rotation. This theory produces equations analogous to Maxwell's electromagnetic equations [3].

In WUM, the World consists of the Medium (protons, electrons, photons, neutrinos, and dark matter particles) and Macroobjects (Galaxy clusters, Galaxies, Star clusters, Extrasolar systems, planets, etc.) made of these particles. There are no empty space and dark energy in the WUM.

Mach's principle. A very general statement of Mach's principle is "Local physical laws are determined by the large-scale structure of the universe".

Fifth dimension. In 1983, Paul S. Wesson suggested that a fifth dimension might be associated with rest mass via $x^{4}=G m / c^{2} \propto t$ [4].

WUM follows this idea, albeit associating the fifth dimension with the parameters of the Medium of the World: the gravitomagnetic parameter and the total energy.

Principal role of Maxwell's Equations (ME) that form the foundation of classical electrodynamics. The value of ME is even greater because J. Swain showed that "linearized general relativity admits a formulation in terms of gravitoelectric and gravitomagnetic fields that closely parallels the description of the electromagnetic field by Maxwell's equations" [5]. Hans Thirring pointed out this analogy in his "On the formal analogy between the basic electromagnetic equations and Einstein's gravity equations in first approximation" paper published in 1918 [6]. It allows us to use formal analogies between the electromagnetism and relativistic gravity. It is worth noting that Oliver Heaviside published the equations for Gravitoelectromagnetism as a separate theory expanding Newton's law as early as 1893, ahead of Einstein's general relativity [7].

Fundamental parameters and units. In accordance with ME, there are two measurable physical characteristics for electromagnetism and gravitoelectromagnetism: energy density and energy flux density. For all particles under consideration, we used four-momentum to conduct statistical analysis of particles' ensembles, obtaining the energy density as the final result.

Two Fundamental parameters in various rational exponents define all macro and micro features of the World: Fine-structure constant $\alpha$ and dimensionless quantity $Q$. While $\alpha$ is constant, $Q$ increases in time, and is in fact a measure of the Size and the Age of the World.

In Section 2 we propose a new physical meaning of the fifth coordinate and give a short summary of WUM. In Section 3 we calculate the parameters of Low Density Intergalactic Plasma that is part of the Medium. Based on the plasma parameters, we calculate Temperature of the Microwave Background Radiation and the rest mass of photons. In Section 4 we point out on decisive role of the Medium of the World composed of massive particles in 5D Space-Time-Energy WUM.

\section{Cosmology}

Let's proceed to discuss the origin, evolution, and parameters of the World speculated by the WUM in light of the Space-Time-Matter theory developed by Paul S. Wesson.

\subsection{The Beginning and Expansion}

The World was started by a fluctuation in the 4-dimensional Universe, and the Nucleus of the World, which is a 4-ball, was born. The Nucleus antipode length (the furthest distance between any two points of the Nucleus 3 -sphere) at the Beginning was equal to $a$.

The Nucleus has since been expanding through the Universe so that the antipode length $R$ is increasing with speed $c$ that is the gravitoelectrodynamic constant, for cosmological time $\tau$ and equals to $R=c \tau$. The corresponding diameter of the Nucleus $D_{N}$ is: $D_{N}=2 R / \pi$. 
The 4-ball is the interior of a 3-sphere which is the World in our Model. The 3-dimensional cubic hyperarea of a 3 -sphere $V_{W}$ is:

$$
V_{W}=\frac{\pi^{2}}{4} D_{N}^{3}=\frac{2}{\pi} R^{3}
$$

Let's introduce a dimensionless time-varying quantity $Q=R / a . Q$ is then the size of the World measured in terms of $a$. The quantity $Q$ is one of the Fundamental parameters of the WUM.

The World consists of the Medium (protons, electrons, photons, neutrinos, and dark matter particles) and Macroobjects (Galaxy clusters, Galaxies, Star clusters, Extrasolar systems, planets, etc.) made of these particles. The WUM is based on Maxwell's equations, and McCullagh's theory [3] is a good fit for description of the Medium.

\subsection{Newtonian Parameter of Gravitation. Primary Parameters of the World}

The (almost) constancy of the universe fundamental constants, including Newtonian constant of gravitation $G$, is now commonly accepted, although has never been firmly established as a fact. All conclusions on the constancy of $G$ are model-dependent [8] [9]. In our opinion, it is impossible to either prove or disprove the constancy of $G$. Consequently, variability of $G$ with time can legitimately be explored. Alternative cosmological models describing the Universe with time varying $G$ are widely discussed in literature (see e.g. [8] [9] and references therein).

A commonly held opinion states that gravity has no established relation to other fundamental forces, so it does not appear possible to calculate it indirectly from other constants that can be measured more accurately, as is done in some other areas of physics. The World-Universe Model holds that there indeed exist relations between all $Q$-dependent, time varying parameters: $G, H$ (Hubble's parameter), $R$ (Size of the World), $A_{\tau}$ (Age of the World), $\rho_{c r}$ (Critical energy density of the World), $T_{\mathrm{MBR}}$ (Temperature of the microwave background radiation).

Recall the well-known Friedmann equation for the critical energy density of the World $\rho_{c r}$ :

$$
\rho_{c r}=\frac{3 H^{2} c^{2}}{8 \pi G}
$$

Equation (2.2.1) can be rewritten as

$$
\frac{4 \pi G}{c^{2}} \times \frac{2}{3} \rho_{c r}=\mu_{g} \times \rho_{M}=H^{2}=\frac{1}{\tau^{2}}=\frac{c^{2}}{R^{2}}
$$

where $\mu_{g}=\frac{4 \pi G}{c^{2}}$ is the gravitomagnetic parameter and $\rho_{M}=\frac{2}{3} \rho_{c r}$ is the energy density of the Medium.

Let's introduce a length parameter $L_{g}$ that is the geometric mean of the Worlds' current size $R$ and its size at the Beginning $a$ :

$$
L_{g}=\sqrt{a R}
$$

In our Model, $L_{g}$ is a convenient basic unit of measure of macroobjects' size. Next, we make a seemingly far-fetched assumption that we will soon show to be in excellent numerical agreement with experimental data:

$$
2 L_{g} L_{P}=a^{2}
$$

which is equivalent to:

$$
D_{N} l_{P}^{2}=D_{N} \frac{\hbar G}{c^{3}}=a_{0}^{3}
$$

where $L_{P}=2 \pi l_{P}$, and $l_{P}$ is Planck length, $\hbar$ is the reduced Planck constant, $a=2 \pi a_{0}$, and $a_{0}$ is the classical electron radius. The size of the World $R$ is then

$$
R=a \times Q=\frac{\pi}{2} D_{N}=\frac{\pi}{2} \frac{a_{0}^{3}}{l_{P}^{2}}=\frac{\pi}{2} \frac{\left(a_{0} c\right)^{3}}{\hbar} \times G^{-1}
$$


According to the Equation (2.2.6), $G$ is proportional to $R^{-1}$ and is decreasing in time as $G \propto \tau^{-1}$. It means that $\rho_{c r}$ and $\rho_{M}$ are also proportional to $R^{-1}$ and are decreasing in time as $\rho_{M}=\frac{2}{3} \rho_{c r} \propto \tau^{-1}$. From (2.2.6), the gravitational parameter $G$ equals to:

$$
G=\frac{\left(a_{0} c\right)^{3}}{4 \hbar a_{0}} \times Q^{-1}
$$

and from (2.2.2), the critical energy density equals to:

$$
\begin{gathered}
\rho_{c r}=3 \rho_{0} \times Q^{-1} \\
\rho_{0}=\frac{h c}{a^{4}}
\end{gathered}
$$

where $\rho_{0}$ is the basic unit of energy density and $h$ is Planck constant. The extrapolated energy density of the World at the Beginning $(Q=1)$ is much smaller than the nuclear energy density. We can now calculate the age of the World $A_{t}$ at current time $t$ :

$$
A_{t}=\frac{R}{c}=\frac{\pi}{2} \frac{\left(a_{0} c\right)^{3}}{\hbar c} \times G^{-1}
$$

Calculating the value of Hubble's parameter $H_{0}$ based on $A_{t}$, we find

$$
H_{0}=\frac{1}{A_{t}}=68.7457(83) \frac{\mathrm{km} / \mathrm{s}}{\mathrm{Mpc}}
$$

which is in good agreement with $H_{0}=69.32 \pm 0.8 \frac{\mathrm{km} / \mathrm{s}}{\mathrm{Mpc}}$ obtained using WMAP data [10]. Close values of calculated and measured parameter $H_{0}$ prove our assumption about the relationship between the basic unit of measure of macroobjects' size $L_{g}$, Plank length $l_{P}$ and the classical electron radius $a_{0}(2.2 .4),(2.2 .5)$. From (2.2.6) we calculate the value of the dimensionless parameter $Q$ :

$$
Q=\frac{\left(a_{0} c\right)^{3}}{4 \hbar a_{0}} \times G^{-1}=0.760000(91) \times 10^{40}
$$

Parameter $Q$ defines both the size and the age of the World measured in terms of $a$ and $t_{0}=a / c$.

In frames of WUM the parameter $G$ can be calculated based on the value of the energy density of the Medium of the World $\rho_{M}$ :

$$
G=\frac{\rho_{M}}{4 \pi} \times P^{2}
$$

where a dimension-transposing parameter $P$ equals to:

$$
P=\frac{a^{3}}{2 h / c}
$$

Then the Newton's law of universal gravitation can be rewritten in the following way:

$$
F=G \frac{m \times M}{r^{2}}=\frac{\rho_{M}}{4 \pi} \frac{\frac{a^{3}}{2 L_{C m}} \times \frac{a^{3}}{2 L_{C M}}}{r^{2}}
$$

where we introduced the measurable parameter of the Medium $\rho_{M}$ instead of the phenomenological coefficient $G$; and gravitoelectromagnetic charges $\frac{a^{3}}{2 L_{C m}}$ and $\frac{a^{3}}{2 L_{C M}}$ instead of macroobjects masses $m$ and $M\left(L_{C m}\right.$ 
and $L_{C M}$ are Compton length of mass $m$ and $M$ respectively). The gravitoelectromagnetic charges have a dimension of "area", which is equivalent to "energy", with the constant that equals to $\sigma_{0}=\rho_{0} a$. Following this approach, we can find the gravitomagnetic parameter of the Medium $\mu_{g}$ :

$$
\mu_{g}=\frac{4 \pi G}{c^{2}}=\frac{1}{R} \times P
$$

and the impedance of the Medium $Z_{g}$ :

$$
Z_{g}=\mu_{g} c=H \times P
$$

We apply the following transformation to Maxwell's equations for the gravitoelectromagnetism: multiply "mass" by the parameter $P$ and divide the impedance and gravitomagnetic parameter of the Medium by the same parameter $P$. As a result of this transformation:

- All parameters of the gravitoelectromagnetic field have dimensions of length and time; "mass" dimension has disappeared;

- All physical parameters of the World measured in terms of $a$ and $t_{0}$ become scalars;

- Absolute size and age of the World equal to $Q$;

- The gravitoelectromagnetic charge has a dimension of "area";

- The impedance of the Medium $Z_{M}$ equals to the Hubble's parameter $H$ for the whole World.

It follows that measuring the value of Hubble's parameter anywhere in the World and taking its inverse value allows us to calculate the absolute age of the World. The Hubble's parameter is then the most important characteristic of the World, as it defines the Worlds' age.

The second important characteristic of the World is the gravitomagnetic parameter $\mu_{M}$ :

$$
\mu_{M}=\frac{1}{R}
$$

Taking its inverse value, we can find the absolute size of the World. We emphasize that the above two parameters $\left(Z_{M}\right.$ and $\left.\mu_{M}\right)$ are principally different physical characteristics of the Medium that are connected through the gravitoelectrodynamic constant $c$.

In WUM, time and space are closely connected with the Mediums' impedance and gravitomagnetic parameter. It follows that neither time nor space could be discussed in absence of the Medium. Matter, then, is primary to time and space. It follows that the gravitational parameter $G$ can be introduced only for the World filled with matter, as Einstein has postulated.

While in our Model Hubble's parameter $H$ has a clear physical meaning, the gravitational parameter $G=\frac{c^{3}}{8 \pi \sigma_{0}} H$ is a phenomenological coefficient in the Newton's law of universal gravitation and in Einstein's theory of general relativity.

\subsection{The Creation of Matter}

Amount of additional surface energy of the 4-ball Nucleus provided by the Universe $\mathrm{d} E_{W}$ is proportional to the increase of the hyperarea of the 3-sphere $V_{W}$ :

$$
\mathrm{d} V_{W}=\frac{6}{\pi} R^{2} \mathrm{~d} R
$$

and the energy density of the Medium $\rho_{M}$ which is the surface energy density of the Nucleus.

The total amount of the surface energy at cosmological time $\tau$ is thus

$$
E_{W}=\frac{12}{\pi} \rho_{0} a \int_{0}^{R} r \mathrm{~d} r=\frac{6}{\pi} \rho_{0} a R^{2}
$$

The energy density of the World $\rho_{W}$ is inversely proportional to the Nucleus antipode length $R$ :

$$
\rho_{W}=\frac{6 \pi^{3} \rho_{0} a R^{2}}{2 \pi^{3} R^{3}}=3 \frac{\rho_{0} a}{R}=\rho_{c r}
$$


and equals to $\rho_{c r}$ necessary for the flat World at any cosmological time $\tau$. It is important to note that in our calculations we used the measurable Fundamental unit-energy density.

All physical parameters under consideration depend on Nucleus diameter $D_{N}$ which is in fact the fifth coordinate in our Model. The quantity $Q$ is the dimensionless value of it.

\subsection{Physical Meaning of the Fifth Coordinate}

According to Overduin and Wesson: "a fifth dimension might be associated with rest mass via $x^{4}=\mathrm{Gm} / \mathrm{c}^{2} \propto t$. The chief effect of this new coordinate on four-dimensional physics was that particle rest mass, usually assumed to be constant, varied with time" [11].

In WUM, the Medium of the World has the following parameters:

- The gravitomagnetic parameter $\mu_{g}=4 \pi G / c^{2} \propto \tau^{-1}$;

- The energy density $\rho_{M} \propto \tau^{-1}$;

- The 3-dimensional cubic hyperarea of a 3-sphere $V_{W}=\frac{\pi^{2}}{4} D_{N}^{3}=\frac{2}{\pi} R^{3} \propto \tau^{3}$

In our opinion, the fifth dimension is associated with these parameters via

$$
x^{4}=\frac{\mu_{g}}{c^{2}} \times \rho_{M} \times V_{W}=\frac{\mu_{g} E_{M}}{c^{2}}=\frac{V_{W}}{R^{2}}=\frac{2}{\pi} R=\frac{2}{\pi} Q a=D_{N} \propto \tau
$$

where $E_{M}=\rho_{M} V_{W}$ is the total surface energy of the 4-ball Nucleus.

\subsection{Experimental Evidence of the Fifth Coordinate}

The physical laws we observe appear to be independent of the fifth coordinate due to the very small value of the dimension-transposing parameter $\mu_{g}$. Then direct observation of the fifth dimension would appear to be a hopeless goal.

One way to prove the existence of the fifth dimension is direct measurement of truly large-scale parameters of the World: Gravitational, Hubble's, Temperature of the Microwave Background Radiation. Conducted at various points of time, these measurements would give us varying results, providing insight into the 5D nature of the World. Unfortunately, the accuracy of the measurements is quite poor. Measurement errors far outweigh any possible $5 \mathrm{D}$ effects, rendering this technique useless in practice. To be conclusive, the measurements would have to be conducted billions of years apart.

Let's consider an effect that has indeed been observed for billions of years, albeit indirectly. Take the socalled "Faint young Sun" paradox. One of the consequences of WUM holds that all stars were fainter in the past. As their cores absorb new matter, the size of macroobjects $R_{M O}$ and their luminosity $L_{M O}$ are increasing in time $R_{M O} \propto Q^{1 / 2} \propto \tau^{1 / 2}$ and $L_{M O} \propto Q \propto \tau$ respectively. Taking the age of the World $\cong 14.2$ Byr and the age of solar system $\cong 4.6 \mathrm{Byr}$, it is easy to find that the young Suns' output was $67 \%$ of what it is today. Literature commonly refers to the value of $70 \%$ [12]. This result supports the notion of physical parameters being indeed dependent on the fifth coordinate.

The proposed approach to the fifth dimension is in agreement with Mach's principle: "Local physical laws are determined by the large-scale structure of the universe". Applied to WUM, it follows that all parameters of the World depending on $Q$ are a manifestation of the fifth dimension of the World. The Medium of the World composed of massive particles is the manifestation of the metric depending on $x^{4}$ [4] [11]. Rest masses of protons, electrons, and Dark Matter particles don't vary with time.

\section{Astroparticle Physics}

In this Section we prove that the Medium of the World consists of massive particles, including photons. We find parameters of the Low Density Intergalactic Plasma, which allow us to calculate the Temperature of the Microwave Background Radiation and the rest mass of photons.

\subsection{Low Density Intergalactic Plasma. Temperature of the Microwave Background Radiation}

In our Model, the World consists of stable massive elementary particles with lifetimes longer than the age of the 
World. Protons with mass $m_{p}$ and energy $E_{p}=m_{p} c^{2}$ and electrons with mass $m_{e}$ and energy $E_{e}=m_{e} c^{2}=\alpha E_{0}$ have identical concentrations in the World: $n_{p}=n_{e}$, where $E_{0}=h c / a$ is the basic energy and $\alpha$ is the finestructure constant.

Low density intergalactic plasma consisting of protons and electrons has plasma frequency $\omega_{p l}$ :

$$
\omega_{p l}^{2}=\frac{4 \pi n_{e} e^{2}}{4 \pi \varepsilon_{0} m_{e}}=4 \pi n_{e} \alpha \frac{h}{2 \pi m_{e} c} c^{2}=2 n_{e} a c^{2}
$$

where $e$ is the elementary charge and $\varepsilon_{0}$ is the permittivity of the Medium.

Let's assume that $\omega_{p l}$ is proportional to $Q^{-1 / 2} \cdot \omega_{p l}^{2}$ is then proportional to $Q^{-1}$. Energy densities of protons and electrons are then proportional to $\frac{1}{R}$, similar to the critical energy density $\rho_{c r} \propto \frac{1}{R}$. Since the formula calculating the potential energy of interaction of protons and electrons contains the same parameter $k_{p e}$ :

$$
k_{p e}=m_{p} \omega_{p l}^{2}=m_{e}\left(\frac{2 \pi c}{L_{g}}\right)^{2}
$$

we substitute $\omega_{p l}^{2}=\frac{m_{e}}{m_{p}}\left(\frac{2 \pi c}{L_{g}}\right)^{2}$ into (3.1.1) and calculate concentration of protons and electrons:

$$
n_{p}=n_{e}=\frac{2 \pi^{2}}{a^{3}} \frac{m_{e}}{m_{p}} \times Q^{-1}
$$

$\rho_{p}=n_{p} E_{p}$ is the energy density of protons in the Medium. The relative energy density of protons $\Omega_{p}$ is then the ratio of $\rho_{p} / \rho_{c r}$ :

$$
\Omega_{p}=\frac{\rho_{p}}{\rho_{c r}}=\frac{2 \pi^{2} \alpha}{3}=0.048014655
$$

The above value is in good agreement with ordinary matter's share in the World $\Omega_{p} \cong 0.049$ found by Planck Collaboration [13].

From Equation (3.1.2) we obtain the value of the lowest radio-wave frequency $v_{p l}$ :

$$
v_{p l}=\frac{\omega_{p l}}{2 \pi}=\left(\frac{m_{e}}{m_{p}}\right)^{1 / 2} \times \frac{c}{a} \times Q^{-1 / 2}=4.5322 \mathrm{~Hz}
$$

Substituting size of the World $R$ obtained in (2.2.6), we use (3.1.3) to calculate the proton and electron concentrations in the Medium:

$$
n_{p}=n_{e}=\frac{1}{\pi} \frac{m_{e}}{m_{p}} \frac{l_{P}^{2}}{a_{0}^{5}}=0.25480 \mathrm{~m}^{-3}
$$

A. Mirizzi, G. G. Raffelt, and P. D. Serpico found that the mean diffuse intergalactic plasma density is bounded by $n_{e} \lesssim 0.27 \mathrm{~m}^{-3}$ [14] corresponding to the WMAP measurement of the baryon density [15]. The Mediums' plasma density $n_{e}=0.25480 \mathrm{~m}^{-3}$ is in good agreement with the measured value and proves the assumption made for plasma frequency: $\omega_{p l} \propto Q^{-1 / 2}$.

The black body spectrum of Microwave Background Radiation (MBR) is due to thermodynamic equilibrium of photons with low density intergalactic plasma consisting of protons and electrons. $\rho_{e}=n_{e} E_{e}$ is the energy density of electrons in the Medium. $\rho_{\mathrm{MBR}}$, the energy density of MBR, equals to twice the value of $\rho_{e}$ :

$$
\rho_{\mathrm{MBR}}=2 \rho_{e}=4 \pi^{2} \alpha \frac{m_{e}}{m_{p}} \rho_{0} \times Q^{-1}=\frac{8 \pi^{5}}{15} \frac{k_{B}^{4}}{(h c)^{3}} T_{\mathrm{MBR}}^{4}
$$


where $k_{B}$ is the Boltzmann constant and $T_{\mathrm{MBR}}$ is MBR temperature. We can now calculate the value of $T_{\mathrm{MBR}}$ :

$$
T_{\mathrm{MBR}}=\frac{E_{0}}{k_{B}}\left(\frac{15 \alpha}{2 \pi^{3}} \frac{m_{e}}{m_{p}}\right)^{1 / 4} \times Q^{-1 / 4}=2.72518 \mathrm{~K}
$$

Thus calculated value of $T_{\mathrm{MBR}}$ is in excellent agreement with experimentally measured value of $2.72548 \pm$ $0.00057 \mathrm{~K}[16]$ and proves the assumption (3.1.7).

At the Beginning of the World, the extrapolated value of $T_{\mathrm{MBR} 0}$ at $Q=1$ is

$$
T_{\text {MBR } 0}=2.1927 \mathrm{MeV}=2.5445 \times 10^{10} \mathrm{~K}
$$

Note that $T_{\mathrm{MBR} 0}$ is considerably smaller than values commonly discussed in literature.

\subsection{Mass Varying Photons. Speed of Light}

Photons with energy smaller than $E_{p h}=h v_{p l}$ cannot propagate in plasma, thus $h v_{p l}$ is the smallest amount of energy a photon may possess. This amount of energy can be viewed as a particle (we'll name it axion), whose frequency-independent effective "rest mass" equals to

$$
m_{a}=\frac{E_{a}}{c^{2}}=\left(\frac{m_{e}}{m_{p}}\right)^{1 / 2} \times m_{0} \times Q^{-1 / 2}=1.8743 \times 10^{-14} \mathrm{eV} / c^{2}
$$

where $E_{a}$ is a rest energy of the axion and $m_{0}$ is a basic unit of mass that equals to: $m_{0}=E_{0} / \mathrm{c}^{2}$.

The calculated mass of an axion is in agreement with $m_{a} \sim 10^{-15} \mathrm{eV} / c^{2}$ discussed by Csaki et al. [17].

According to special relativity, energy of an axion $E_{a}$ moving with a group velocity $v_{g r}$ is given by

$$
E_{a}\left(v_{g r}\right)=h v_{p l}\left(1-\frac{v_{g r}^{2}}{c^{2}}\right)^{-1 / 2}
$$

Taking into account the dispersion relation for plasma:

$$
v_{g r} v_{p h}=c^{2}
$$

and the value of phase velocity $v_{p h}=\frac{c}{n_{p l}}$, where $n_{p l}$ is the index of plasma refraction:

$$
n_{p l}=\left(1-\frac{v_{p l}^{2}}{v^{2}}\right)^{1 / 2}
$$

we calculate moving axion energy $E_{a}\left(v_{g r}\right)$ to be

$$
E_{a}\left(v_{g r}\right)=h v=E_{p h}
$$

where $v$ is photon frequency. In our Model, the total energy of a moving particle consists of two components: rest energy and constituent energy. A particles' constituent energy is the response of the Medium to the particles' movement.

A photon is then a constituent axion with rest energy $E_{a}=h v_{p l}$ and total energy $E_{p h}=h v$. In most cases $v \gg v_{p l}$, and practically all of the photons' energy is the axions' constituent energy. Axions are fully characterized by their four-momentum. Rest energy of the axion is decreasing with time: $E_{a} \propto t^{-1 / 2}$ (see (3.2.1)).

The higher the photons' energy, the closer its speed approaches $c$. But the fact that axions possess non-zero rest masses means that photons can never reach that speed. It is worth to note that the speed of light in vacuum, commonly denoted $c$, is not related to the World in our Model, because there is no vacuum in it. Instead, there is the Medium of the World consisting of elementary particles. 


\section{Medium of the World}

J. M. Overduin and P. S. Wesson postulated that "Metrics which do not depend on $x^{4}$ can give rise only to induced matter composed of (massless) photons; while those which depend on $x^{4}$ give back equations of state for fluids composed of massive particles" [11].

The World-Universe Model supplies the fluid that J. M. Overduin and P. S. Wesson have predicted: it is, in fact, the Medium of the World. According to WUM, empty space does not exist; instead, the World is filled with Medium that consists of massive particles: protons, electrons, photons, neutrinos, and dark matter particles. The inter-galactic voids discussed by astronomers are in fact examples of the Medium in its purest. Consequently, the Medium of the World as described by WUM can serve as further evidence in favor of the fifth-dimensional view of the World.

\section{Conclusion}

5D Space-Time-Energy World-Universe Model is the unified model of the World around the concept of Medium that successfully describes all of the primary parameters and their relationships. The Model allows for precise calculation of values that is only measured experimentally earlier: Hubble's parameter, low density intergalactic plasma parameters, temperature of microwave background radiation and the rest mass of photons. While the Model needs significant further elaboration, it can already serve as a basis for a new physics proposed by Paul Dirac in 1937 and Paul Wesson in 1983.

\section{Acknowledgements}

I am very grateful to Prof. Paul S. Wesson for the development of Space-Time-Matter theory. Many thanks to Pete Carroll, William Straub and Nainan Varghese for our stimulating correspondence; Felix Lev and my son Ilya Netchitailo for valuable discussions.

\section{References}

[1] Dirac, P.A.M. (1937) The Cosmological Constants. Nature, 139, 323. http://dx.doi.org/10.1038/139323a0

[2] Hoyle, F. and Narlikar, J.V. (1964) A New Theory of Gravitation. Proceedings of the Royal Society of London, A282, 178. http://dx.doi.org/10.1098/rspa.1964.0227

[3] McCullagh, J. (1846) An Essay towards a Dynamical Theory of Crystalline Reflexion and Refraction. Transactions of the Royal Irish Academy, 21, 17.

[4] Wesson, P.S. (1983) A New Approach to Scale-Invariant Gravity. Astronomy Astrophysics, 119, 145.

[5] Swain, J. (2010) Gravitatomagnetic Analogs of Electric Transformers. arXiv: ge-qc/1006.5754v1.

[6] Thirring, H. (1918) On the Formal Analogy between the Basic Electromagnetic Equations and Einstein's Gravity Equations in First Approximation. Physikalische Zeitschrift, 19, 204.

[7] Heaviside, O. (1893) A Gravitational and Electromagnetic Analogy. The Electrician, 31, 81.

[8] Uzan, J.P. (2002) The Fundamental Constants and Their Variation: Observational Status and Theoretical Motivations. arXiv: hep-ph/0205340v1.

[9] Uzan, J.P. (2011) Varying Constants, Gravitation and Cosmology. Living Reviews in Relativity, 14, 2.

[10] Bennett, C.L., et al. (2013) Nine-Year Wilkinson Microwave Anisotropy Probe (WMAP) Observations: Final Maps and Results. arXiv: astro-ph/1212.5225v3.

[11] Overduin, J.M. and Wesson, P.S. (1998) Kaluza-Klein Gravity. http://arxiv.org/abs/gr-qc/9805018

[12] Gough, D.O. (1981) Solar Interior Structure and Luminosity Variations. Solar Physics, 74, 21-34. http://dx.doi.org/10.1007/BF00151270

[13] Matthew, F. (2013) First Planck Results: The Universe Is Still Weird and Interesting. http://arstechnica.com/science/2013/03/first-planck-results-the-universe-is-still-weird-and-interesting/

[14] Mirizzi, A., Raffelt, G.G. and Serpico, P.D. (2006) Photon-Axion Conversion in Intergalactic Magnetic Fields and Cosmological Consequences. http://arxiv.org/abs/astro-ph/0607415

[15] Spergel, D.N., Verde, L., Peiris, H.V., Komatsu, E., Nolta, M.R., Bennett, C.L., et al. (2003) First Year Wilkinson Microwave Anisotropy Probe (WMAP) Observations: Determination of Cosmological Parameters. 
http://arxiv.org/abs/astro-ph/0302209

[16] Fixsen, D.J. (2009) The Temperature of the Cosmic Microwave Background. http://arxiv.org/abs/0911.1955

[17] Csaki, C., Kaloper, N. and Terning, J. (2001) Effects of the Intergalactic Plasma on Supernova Dimming via Photon-Axion Oscillations. http://arxiv.org/abs/hep-ph/0112212 